\title{
Wave Number and Input Impedance of a VLF Insulated Linear Antenna in an Anisotropic Ionosphere
}

\author{
Shitian Zhang, ${ }^{1,2}$ Huaiyun Peng, ${ }^{2}$ Bing Wei, ${ }^{1}$ Xiange Han, ${ }^{1}$ and Maoyan Wang ${ }^{3}{ }^{3}$ \\ ${ }^{1}$ School of Physics and Optoelectronic Engineering, Xidian University, Xi'an, China \\ ${ }^{2}$ National Key Laboratory of Electromagnetic Environment, Qingdao, China \\ ${ }^{3}$ School of Physics, University of Electronic Science and Technology of China, Chengdu, China \\ Correspondence should be addressed to Maoyan Wang; wmybrimlhl@163.com
}

Received 28 June 2021; Accepted 23 October 2021; Published 3 November 2021

Academic Editor: Mohammad Alibakhshikenari

Copyright (c) 2021 Shitian Zhang et al. This is an open access article distributed under the Creative Commons Attribution License, which permits unrestricted use, distribution, and reproduction in any medium, provided the original work is properly cited.

We report a method to obtain the wave number and input impedance of a very low frequency (VLF) insulated linear antenna in an anisotropic ionosphere. Due to the anisotropy, electromagnetic fields in the ionosphere are decomposed into the ordinary wave and extraordinary wave. Wave equations for the layered structure are applied to access the wave number of the insulated antenna in the ionosphere via the derivation of the eigenvalue equation by using boundary conditions. The expression for the wave number is given based on some approximation formulas. Then, King's antenna theory is further employed to solve the input impedance and current distribution of the antenna in the anisotropic medium. After the validation of the method is performed, near-field characteristics for an insulated antenna with different medium parameters in the anisotropic ionosphere are discussed. Effects of the electric density and geomagnetic field of the time-and space-varying anisotropic ionosphere on the distribution of normalized current are analyzed. This finding provides a promising avenue for getting electromagnetic characteristics of spaceborne antennas.

\section{Introduction}

The very low-frequency (VLF) $(3-30 \mathrm{kHz})$ antennas play an important role in the underwater communication, navigation, ionosphere, and earthquake detection [1, 2]. The trailing antenna, loop antenna, and antenna array are common VLF transmitting antennas [3-6]. Most of VLF antenna arrays, which are expensive and easily damaged due to natural disasters, were built on large ground-based transmitting stations. Since the United States space shuttle Columbia successfully carried out the tethered satellite system release test [3] and VLF signal was received on the ground, the space-borne VLF communication system is becoming more and more possible [7, 8]. The tethered satellites with VLF antennas, which are operated on a low earth orbit of $300-400 \mathrm{~km}$ from the Earth, are generally placed in the F2 layer of the ionosphere [4]. Because the satellites fly around the Earth, the transmitted VLF electromagnetic waves can cover major regions without great power loss. However, theoretical works on the VLF antennas are mainly based on experiments; the anisotropy of the ionosphere is nearly considered in these works.

The radiations from various sources in a magnetoionic medium have been studied [9-15]. Wang and Bell give approximate closed form expressions for the radiation resistance of an arbitrarily oriented electric dipole antenna in a cold magnetoplasma $[16,17]$. The absence of the insulation layer makes the radiation efficiency of the bare antenna be seriously reduced because the ionosphere is a medium with high conductivity [18]. In 1973, Wu et al. studied properties of an insulated dipole antenna if the wavenumber of the surrounding infinite, homogeneous, and isotropic medium is larger than that of the insulation layer [19]. Balmain puts forward a formula for the impedance of a short cylindrical dipole in a magnetoplasma by using the quasi-static electromagnetic theory [20]. In 2001, Nikitin and Swenson presented the analysis of the impedance of a short dipole antenna in a cold plasma by using a quasi-static approach. 
Either the anisotropy was not considered or influences of the insulation layer were neglected in these studies [21]. Chen and Seshadri investigated characteristics of an infinite insulated cylindrical antenna in a simple uniaxially anisotropic medium [22]. Galejs developed an avariational formula for the impedance of a finite insulated cylindrical antenna of length $\lambda / 20$ and $\lambda / 10$ in a cold plasma with a longitudinal magnetic field, where $\lambda$ is the free space wavelength [23]. Because the radiation capability of a linear antenna is dependent on the total current moment, the radiation power of such an electrically small antenna is not enough. The electric density and geomagnetic field of the F2 layer vary a little with the height; the current distribution along a VLF antenna is mainly affected by its near-field. However, available works are focused on the radiation impedance and power instead of the wave number and input impedance. The research on the interaction mechanism between VLF electromagnetic waves and an insulated linear antenna in the anisotropic ionosphere, which can be used to adjust the antenna performance, becomes necessary. The performance of an insulated linear antenna placed in the same height of the ionosphere influenced by the day, night, longitude, and latitude is addressed in this paper.

Moreover, analytical and numerical methods used to simulate the wave number and input impedance of a VLF insulated linear antenna in the anisotropic ionosphere are desirable. In 2005, Kudrin et al. discussed characteristics of a good conducting cylindrical antenna insulated from the surrounding cold collision less magnetoplasma by an isotropic coaxial cylindrical sheath [24]. In 2019, He et al. analytically studied the current distribution and input impedance of a VLF linear bare antenna in an anisotropic plasma $[5,25]$. In 2020 , Zeng et al. analytically researched effects of the insulation thickness on the current distribution and input impedance of an insulated linear antenna in an anisotropic plasma [26]. In 2021, Bouknia et al. theoretically studied the input impedance and electromagnetic field distribution of a dipole antenna printed on an electrical/ magnetic uniaxial anisotropic substrate by using the method of moments [27]. The introduction of the anisotropy makes the electric field and electric current of an insulated antenna in the anisotropic ionosphere very complex and difficult to solve [5, 24-30]. Therefore, King's antenna theory [18] is employed and developed in this paper.

In this paper, near-field characteristics of an insulated linear antenna in an anisotropic ionosphere are studied. The electromagnetic waves, which are expressed in the combination of the ordinary wave (O-wave) and extraordinary wave (E-wave), in the anisotropic ionosphere are given. The eigenvalue equation containing the wave number of the layered antenna structure is derived by using boundary conditions. Then, King's antenna theory is applied to get the input impedance and current distribution of the insulated antenna after the approximate formula for the wave number is obtained. The effects of medium parameters of the insulated antenna and anisotropic ionosphere on the wave number, input impedance, and current distribution of the insulated linear antenna in the ionosphere are investigated.

\section{Theory}

2.1. Wave Equations for an Insulated Antenna in an Anisotropic Ionosphere. The inhomogeneous structure of an insulated antenna in an anisotropic ionosphere is shown in Figure 1. The antenna consists of a good conductor in region I and an insulation layer in region II. $a$ and $b$ are outer radii of the conductor and insulation layer, respectively. The permeability of each region $\mu$ equals to that of free space $\mu_{0}$. The real part of relative permittivity, conductivity, and wave vector for the conductor are $\varepsilon_{I}, \sigma_{I}$, and $k_{\mathrm{I}}=\omega\left[\mu \varepsilon_{0}\left(\varepsilon_{\mathrm{I}}+i \sigma_{\mathrm{I}} / \omega\right)\right]$ $1 / 2 \approx\left(i \omega \mu \varepsilon_{0} \sigma_{\mathrm{I}}\right)^{1 / 2}$ and for the insulation layer are $\varepsilon_{\mathrm{II}}, \sigma_{\mathrm{II}}$, and $k_{\mathrm{II}}=\omega\left[\mu \varepsilon_{0}\left(\varepsilon_{\mathrm{II}}+\mathrm{i} \sigma_{\mathrm{II}} / \omega\right)\right]^{1 / 2}$, respectively. The medium in the IIIrd region is the anisotropic ionosphere. The length of the antenna is $2 l$. The antenna is parallel to the geomagnetic field with the amplitude $B_{0}$. The antenna is driven by applying feeding voltage $V_{0}$, which denotes the Dirac function $\delta(z)$ at its center across a thin gap to generate electromagnetic fields.

The geomagnetic field is assumed to be polarized along $z$ axis. The anisotropic plasma can be described by the permittivity tensor $[23,31,32]$ :

$$
\overline{\bar{\varepsilon}}=\left[\begin{array}{ccc}
\varepsilon_{1} & -i \varepsilon_{2} & 0 \\
i \varepsilon_{2} & \varepsilon_{1} & 0 \\
0 & 0 & \varepsilon_{3}
\end{array}\right],
$$

with

$$
\begin{aligned}
\varepsilon_{1} & =1-X U /\left(U^{2}-Y^{2}\right), \\
\varepsilon_{2} & =X Y /\left(U^{2}-Y^{2}\right), \\
\varepsilon_{3} & =1-X / U \\
U & =1+i v / \omega \\
X & =\omega_{0}^{2} / \omega^{2} \\
Y & =\omega_{H} / \omega \\
\omega_{0}^{2} & =N_{0} e^{2} / m_{e} \varepsilon_{0} \\
\omega_{H} & =\left|e B_{0} / m_{e}\right|
\end{aligned}
$$

where $\omega_{H}$ is the gyromagnetic frequency, $\omega_{0}$ represents the electron cyclotron frequency, $v$ is the collision frequency of electrons, and $\varepsilon_{0}$ is the permittivity of free space.

The Maxwell equations for the ionospheric plasma are

$$
\begin{aligned}
& \nabla \times E=i \omega B, \\
& \nabla \times B=-i \omega \mu \overline{\bar{\varepsilon}} E .
\end{aligned}
$$

In a cylindrical coordinate system, $ə / \partial \phi=0$ due to symmetry. Thus, the differential form of wave equations for the anisotropic ionosphere in the IIIrd region can be expressed as [23] 


$$
\begin{aligned}
\frac{\partial E_{\mathrm{III} \phi}}{\partial z} & =-i \omega \mu H_{\mathrm{III} \rho}, \\
\frac{\partial H_{\mathrm{III} \phi}}{\partial z} & =i \omega\left(\varepsilon_{1} E_{\mathrm{III} \rho}-i \varepsilon_{2} E_{\mathrm{III} \phi}\right), \\
\frac{1}{\rho} \frac{\partial}{\partial \rho}\left(\rho E_{\mathrm{III} \phi}\right) & =i \omega \mu H_{\mathrm{III} z}, \\
\frac{1}{\rho} \frac{\partial}{\partial \rho}\left(\rho H_{\mathrm{III} \phi}\right) & =-i \omega \varepsilon_{3} E_{\mathrm{III} z}, \\
\frac{\partial E_{\mathrm{II} \rho}}{\partial z}-\frac{\partial E_{\mathrm{III} z}}{\partial \rho} & =i \omega \mu H_{\mathrm{III} \phi}, \\
\frac{\partial H_{\mathrm{III} \rho}}{\partial z}-\frac{\partial H_{\mathrm{III} z}}{\partial \rho} & =-i \omega\left(i \varepsilon_{2} E_{\mathrm{III} \rho}+\varepsilon_{1} E_{\mathrm{III} \phi}\right) .
\end{aligned}
$$

In order to efficiently solve (5), a Fourier transform pair is defined as follows:

$$
\begin{aligned}
& F(z)=\frac{1}{2 \pi} \int_{-\infty}^{\infty} F(\zeta) e^{-i \zeta z} \mathrm{~d} \zeta \\
& F(\zeta)=\int_{-\infty}^{\infty} F(z) e^{i \zeta z} \mathrm{~d} z .
\end{aligned}
$$

By applying the Fourier transform to (5) and expressing $E_{I I I \phi}, E_{I I I \rho}, H_{I I I \phi}$, and $H_{I I I \rho}$ in $E_{I I I z}$ and $H_{I I I z}$, the $\phi$ - and $\rho$-components of electromagnetic fields in (5) can be reorganized as

$$
\begin{gathered}
E_{\mathrm{III} \phi}=-\frac{1}{\left(k_{1}^{2}-\zeta^{2}\right)^{2}-k_{2}^{4}}\left[k_{2}^{2} \zeta \frac{\partial E_{\mathrm{III} z}}{\partial \rho}+i \omega \mu\left(k_{1}^{2}-\zeta^{2}\right) \frac{\partial H_{\mathrm{III} z}}{\partial \rho}\right], \\
H_{\mathrm{III} \phi}=\frac{1}{\left(k_{1}^{2}-\zeta^{2}\right)^{2}-k_{2}^{4}}\left[i \frac{k_{1}^{2}\left(k_{1}^{2}-\zeta^{2}\right)-k_{2}^{4}}{\omega \mu} \frac{\partial E_{\mathrm{III} z}}{\partial \rho}-k_{2}^{2} \zeta \frac{\partial H_{\mathrm{III} z}}{\partial \rho}\right], \\
E_{\mathrm{III} \rho}=\frac{1}{\left(k_{1}^{2}-\zeta^{2}\right)^{2}-k_{2}^{4}}\left[-i \zeta\left(k_{1}^{2}-\zeta^{2}\right) \frac{\partial E_{\mathrm{III} z}}{\partial \rho}+\omega \mu k_{2}^{2} \frac{\partial H_{\mathrm{III} z}}{\partial \rho}\right], \\
H_{\mathrm{III} \rho}=\frac{\zeta}{\omega \mu} \frac{1}{\left(k_{1}^{2}-\zeta^{2}\right)^{2}-k_{2}^{4}}\left[-k_{2}^{2} \zeta \frac{\partial E_{\mathrm{III} z}}{\partial \rho}-i \omega \mu\left(k_{1}^{2}-\zeta^{2}\right) \frac{\partial H_{\mathrm{III} z}}{\partial \rho}\right] .
\end{gathered}
$$

The $z$-component of the electric field satisfies

$$
\begin{aligned}
& \frac{\partial^{4} E_{\mathrm{III} z}}{\partial \rho^{4}}+\frac{2}{\rho} \frac{\partial^{3} E_{\mathrm{III} z}}{\partial \rho^{3}}+\left[\frac{\left(k_{1}^{2}+k_{3}^{2}\right)\left(k_{1}^{2}-\zeta^{2}\right)-k_{2}^{4}}{k_{1}^{2}}-\frac{1}{\rho^{2}}\right] \frac{\partial^{2} E_{\mathrm{III} z}}{\partial \rho^{2}} \\
& +\left[\frac{\left(k_{1}^{2}+k_{3}^{2}\right)\left(k_{1}^{2}-\zeta^{2}\right)-k_{2}^{4}}{k_{1}^{2} \rho}+\frac{1}{\rho^{3}}\right] \frac{\partial E_{\mathrm{III} z}}{\partial \rho}+\frac{k_{3}^{2}}{k_{1}^{2}}\left[\left(k_{1}^{2}-\zeta^{2}\right)^{2}-k_{2}^{4}\right] E_{\mathrm{III} z}=0,
\end{aligned}
$$

where

$$
\begin{aligned}
& k_{1}^{2}=\omega^{2} \mu \varepsilon_{0} \varepsilon_{1}, \\
& k_{2}^{2}=\omega^{2} \mu \varepsilon_{0} \varepsilon_{2}, \\
& k_{3}^{2}=\omega^{2} \mu \varepsilon_{0} \varepsilon_{3} .
\end{aligned}
$$

2.2. Boundary Conditions and Eigenvalue Equation. The electromagnetic fields excited in an infinite homogeneous anisotropic medium can be decomposed into the $\mathrm{O}$-wave and E-wave [31, 33]. In the near-field region, both the ordinary wave (O-wave) and extraordinary wave (E-wave) exist. In the far field region, the $\mathrm{O}$-wave coexists with $\mathrm{E}$-wave 
and the interference phenomenon occurs for high-frequency waves. For VLF waves, only the E-wave, which is also called a whistler-mode wave $[34,35]$, exists in the far field region because the $\mathrm{O}$-wave is an evanescent wave. There is a propagation region. The size of the propagation region relates to the working frequency. The smaller the frequency is, the larger the propagation area is. In the propagating region, the radiation directivity is sensitive to the direction of the dipole and geomagnetic field.

The influences of the evanescent O-wave cannot be neglected if near-field effects of the VLF antenna in an anisotropic ionosphere are discussed. The E-wave is a propagable mode with a small attenuation rate as long as the propagation direction is within the critical angle. The wave numbers for the $\mathrm{O}$-wave and E-wave are [33]

$$
k_{o, e}^{2}=\frac{-\left[\left(k_{2}^{4}-k_{1}^{4}\right) \sin ^{2} \theta-k_{1}^{2} k_{3}^{2}\left(1+\cos ^{2} \theta\right)\right] \pm \sqrt{\left(k_{1}^{4}-k_{2}^{4}-k_{1}^{2} k_{3}^{2}\right)^{2} \sin ^{4} \theta+4 k_{2}^{4} k_{3}^{4} \cos ^{2} \theta}}{2\left(k_{1}^{2} \sin ^{2} \theta+k_{3}^{2} \cos ^{2} \theta\right)},
$$

where $\theta$ is the angle between the geomagnetic field and $z$ axis. The electric fields in the $j$ th region can be written as

$$
\begin{gathered}
E_{I z}(\rho, \zeta)=F J_{0}\left(\rho \xi_{2 \mathrm{I}}\right), \quad 0 \leq \rho \leq a, \\
E_{\mathrm{II} z}(\rho, \zeta)=A H_{0}^{(1)}\left(\rho \xi_{\mathrm{II}}\right)+B J_{0}\left(\rho \xi_{\mathrm{II}}\right), \quad a_{+} \leq \rho \leq b, \\
E_{\mathrm{III} z}(\rho, \zeta)=C H_{0}^{(1)}\left(\rho \xi_{o}\right)+D H_{0}^{(1)}\left(\rho \xi_{e}\right), \quad b_{+} \leq \rho \leq \infty,
\end{gathered}
$$

with

$$
\begin{aligned}
& \xi_{\mathrm{I}}=\sqrt{k_{I}^{2}-\zeta^{2}}, \\
& \xi_{\mathrm{II}}=\sqrt{k_{\mathrm{II}}^{2}-\zeta^{2}}, \\
& \xi_{o}=\sqrt{k_{o}^{2}-\zeta^{2}}, \\
& \xi_{e}=\sqrt{k_{e}^{2}-\zeta^{2}}, \\
& k_{\mathrm{I}}=\left(i \omega u \sigma_{\mathrm{I}}\right)^{1 / 2}, \\
& k_{\mathrm{II}}=\omega\left[\mu\left(\frac{\varepsilon_{\mathrm{II}}+i \sigma_{\mathrm{II}}}{\omega}\right)\right]^{1 / 2} .
\end{aligned}
$$

$$
\begin{aligned}
H_{\mathrm{II} \phi}(b, \zeta)= & \frac{i k_{\mathrm{II}}^{2}}{\omega u \xi_{\mathrm{II}}^{2}} \frac{\partial E_{\mathrm{II} z}(b, \zeta)}{\partial \rho}, \\
H_{\mathrm{III} \phi}= & \left\{\frac{k_{1}^{2} \xi_{o}^{2}}{\left[\left(k_{1}^{2}-\zeta^{2}\right)^{2}-k_{2}^{4}\right]}-\left[\frac{k_{1}^{2}\left(k_{1}^{2}-\zeta^{2}\right)-k_{2}^{4}}{\left(k_{1}^{2}-\zeta^{2}\right)^{2}-k_{2}^{4}}+\frac{k_{3}^{2}\left(k_{1}^{2}-\zeta^{2}\right)}{\left[\left(k_{1}^{2}-\zeta^{2}\right)^{2}-k_{2}^{4}\right]}\right]\right\} \frac{i C \xi_{o} H_{1}^{(1)}\left(\rho \xi_{o}\right)}{\omega \mu} \\
& +\left\{\frac{k_{1}^{2} \xi_{e}^{2}}{\left[\left(k_{1}^{2}-\zeta^{2}\right)^{2}-k_{2}^{4}\right]}-\left[\frac{k_{1}^{2}\left(k_{1}^{2}-\zeta^{2}\right)-k_{2}^{4}}{\left(k_{1}^{2}-\zeta^{2}\right)^{2}-k_{2}^{4}}+\frac{k_{3}^{2}\left(k_{1}^{2}-\zeta^{2}\right)}{\left[\left(k_{1}^{2}-\zeta^{2}\right)^{2}-k_{2}^{4}\right]}\right] \frac{i D \xi_{e} H_{1}^{(1)}\left(\rho \xi_{e}\right)}{\omega \mu} .\right.
\end{aligned}
$$

In (18), $k_{I}$ and $k_{I I}$ are wave vectors of electromagnetic fields in regions I and II, respectively.

The boundary condition at $\rho=a$ is

$$
E_{\mathrm{I} z}\left(a_{-}, z\right)=E_{\mathrm{II} z}\left(a_{+}, z\right)=V_{0} \delta(z),
$$

where $\delta(z)$ is the Dirac delta function whose Fourier transform is 1 . One can obtain

$$
\begin{aligned}
E_{\mathrm{I} z}\left(a_{-}, \zeta\right)=E_{\mathrm{II} z}\left(a_{+}, \zeta\right) & =V_{0}, \\
\frac{i k_{\mathrm{II}}^{2}}{\omega u \xi^{2}}\left(\frac{\partial E_{\mathrm{II} z}\left(a_{+}, \zeta\right)}{\partial \rho}-\frac{\partial E_{\mathrm{I} z}\left(a_{-}, \zeta\right)}{\partial \rho}\right) & =\frac{I(\zeta)}{2 \pi a},
\end{aligned}
$$

where $I(\zeta)$ is the transform of $I(z)=2 \pi a J(z)$ and $J(z)$ is the surface current density on the insulated antenna.

Hence, we obtain

$$
A H_{0}^{(1)}\left(a \xi_{\text {II }}\right)+B J_{0}\left(a \xi_{\text {II }}\right)=V_{0} .
$$

The boundary conditions at $\rho=b$ are

$$
\begin{aligned}
E_{\mathrm{II} z}(b, \zeta) & =E_{\mathrm{III} z}(b, \zeta), \\
H_{\mathrm{II} \phi}(b, \zeta) & =H_{\mathrm{III} \phi}(b, \zeta),
\end{aligned}
$$

with 
By substituting (16) and (17) into (23), one can obtain $A H_{0}^{(1)}\left(b \xi_{\mathrm{II}}\right)+B J_{0}\left(b \xi_{\mathrm{II}}\right)-C H_{0}^{(1)}\left(b \xi_{o}\right)-D H_{0}^{(1)}\left(b \xi_{e}\right)=0$.
The following equation can be obtained by substituting (16) into (25), as well as (25) and (26) into (24):

$$
\begin{aligned}
& \frac{k_{\mathrm{II}}^{2}}{\xi_{\mathrm{II}}}\left[A H_{1}^{(1)}\left(b \xi_{\mathrm{II}}\right)+B J_{1}\left(b \xi_{\mathrm{II}}\right)\right]-\left\{\frac{k_{1}^{2} \xi_{o}^{2}}{\left(k_{1}^{2}-\zeta^{2}\right)^{2}-k_{2}^{4}}-\left[\frac{k_{1}^{2}\left(k_{1}^{2}-\zeta^{2}\right)-k_{2}^{4}}{\left(k_{1}^{2}-\zeta^{2}\right)^{2}-k_{2}^{4}}+\frac{k_{3}^{2}\left(k_{1}^{2}-\zeta^{2}\right)}{\left(k_{1}^{2}-\zeta^{2}\right)^{2}-k_{2}^{4}}\right]\right\} \\
& \quad \times C \xi_{o} H_{1}^{(1)}\left(b \xi_{o}\right)-\left\{\frac{k_{1}^{2} \xi_{e}^{2}}{\left(k_{1}^{2}-\zeta^{2}\right)^{2}-k_{2}^{4}}-\left[\frac{k_{1}^{2}\left(k_{1}^{2}-\zeta^{2}\right)-k_{2}^{4}}{\left(k_{1}^{2}-\zeta^{2}\right)^{2}-k_{2}^{4}}+\frac{k_{3}^{2}\left(k_{1}^{2}-\zeta^{2}\right)}{\left(k_{1}^{2}-\zeta^{2}\right)^{2}-k_{2}^{4}}\right] D \xi_{e} H_{1}^{(1)}\left(b \xi_{e}\right)=0 .\right.
\end{aligned}
$$

By substituting (17) into (12), we obtain

$$
\left[k_{3}^{2}\left(k_{1}^{2}-\zeta^{2}\right)-k_{1}^{2} \xi_{o}^{2}\right] C H_{0}^{(1)}\left(b \xi_{o}\right)+\left[k_{3}^{2}\left(k_{1}^{2}-\zeta^{2}\right)-k_{1}^{2} \xi_{e}^{2}\right] D H_{0}^{(1)}\left(b \xi_{e}\right)=0
$$

By using (22), (27), (28), and (29), one can derive homogeneous equations containing field coefficients, i.e.,

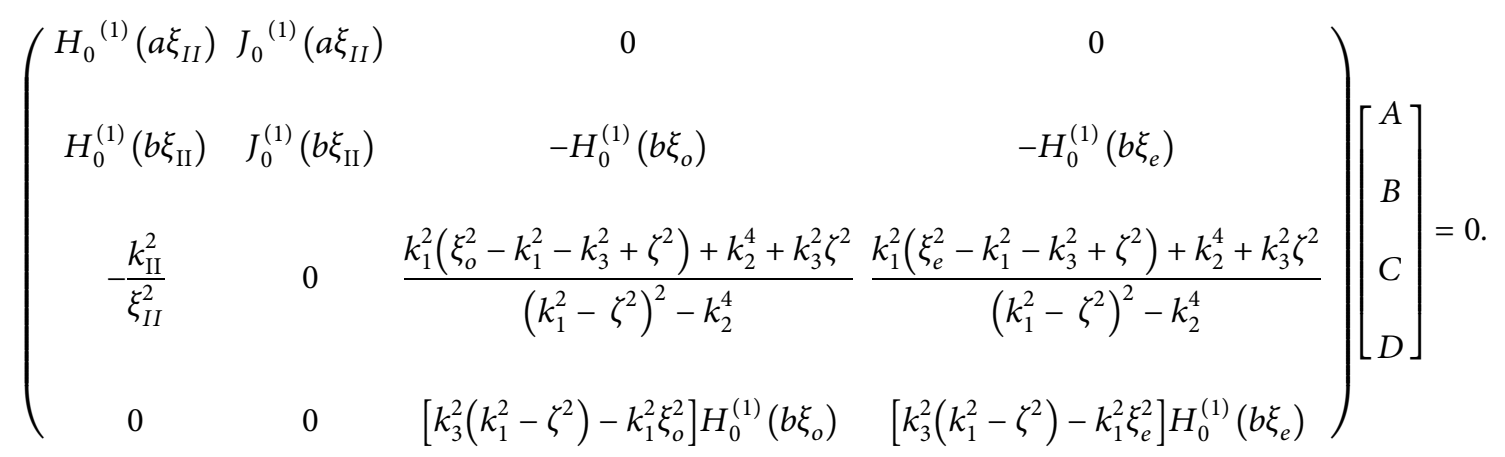

In (30), a solution with nonzero $E$ is possible only if the determinant of the $4 \times 4$ matrix must be zero. After some manipulations, we can find a complicated eigenvalue equation, which can be solved by using some approximations based on propagation characteristics of the VLF electromagnetic waves. The current on the insulted antenna can be further obtained after solving the field coefficients and (21).
2.3. Wave Number. The small collision frequency can be ignored in the ionospheric F-region. The wave vectors in the anisotropic ionosphere satisfy [33]

$$
\begin{aligned}
k_{1}^{2} & \ll k_{2}^{2} \ll k_{3}^{2}, \\
k_{3}^{2} k_{1}^{2} & =-k_{2}^{4} .
\end{aligned}
$$

Approximate expressions for the Bessel and Hank functions can be seen in [18]. If $\zeta=O\left(k_{\mathrm{II}}\right)$ and $\zeta$ approaches $k_{\mathrm{II}}$, then $\xi_{\mathrm{II}}=\sqrt{k_{\mathrm{II}}^{2}-\zeta^{2}}=O\left(k_{\mathrm{II}}\right)$. The eigenvalue equation from the determinant of (30) can be reorganized as

$$
\begin{aligned}
& k_{2}^{4} \ln \frac{a}{b} \frac{k_{\mathrm{II}}^{2}}{\xi_{\mathrm{II}}^{2}} k_{1}^{2}\left(\xi_{e}^{2}-\xi_{o}^{2}\right) H_{0}^{(1)}\left(b \xi_{o}\right) H_{0}^{(1)}\left(b \xi_{e}\right) \\
& =\left[k_{3}^{2} k_{1}^{2}-k_{1}^{2} \xi_{e}^{2}\right]\left(k_{1}^{2} \xi_{o}^{2}-2 k_{3}^{2} k_{1}^{2}\right) H_{0}^{(1)}\left(b \xi_{e}\right)-\left[k_{3}^{2} k_{1}^{2}-k_{1}^{2} \xi_{o}^{2}\right]\left(k_{1}^{2} \xi_{e}^{2}-2 k_{3}^{2} k_{1}^{2}\right) H_{0}^{(1)}\left(b \xi_{o}\right) .
\end{aligned}
$$


(32) can be rewritten as

$$
\zeta^{2}=k_{\mathrm{II}}^{2}\left\{1-\frac{i \pi\left(\xi_{o}^{2}-\xi_{e}^{2}\right) H_{0}^{(1)}\left(b \xi_{o}\right) H_{0}^{(1)}\left(b \xi_{e}\right)}{2 \ln b / a k_{1}^{2} / k_{2}^{4}\left[\left(k_{3}^{2}-\xi_{e}^{2}\right)\left(\xi_{o}^{2}-2 k_{3}^{2}\right) H_{0}^{(1)}\left(b \xi_{e}\right)-\left(k_{3}^{2}-\xi_{o}^{2}\right)\left(\xi_{e}^{2}-2 k_{3}^{2}\right) H_{0}^{(1)}\left(b \xi_{o}\right)\right]}\right\},
$$

where $k_{\mathrm{II}} \ll k_{o}, \xi_{o}=\sqrt{k_{o}^{2}-\zeta^{2}} \approx \stackrel{o}{k}$, and $\xi_{e}=\sqrt{k_{e}^{2}-\zeta^{2}} \approx \stackrel{e}{e}$. If the wave number of the insulated antenna is written as $k_{l}$, that is, $k_{l}=\zeta$, we obtain

$$
k_{l}^{2}=k_{\mathrm{II}}^{2}\left\{1-\frac{i \pi\left(k_{o}^{2}-k_{e}^{2}\right) H_{0}^{(1)}\left(b k_{o}\right) H_{0}^{(1)}\left(b k_{e}\right)}{2 \ln b / a k_{1}^{2} / k_{2}^{4}\left[\left(k_{3}^{2}-k_{e}^{2}\right)\left(k_{o}^{2}-2 k_{3}^{2}\right) H_{0}^{(1)}\left(b k_{e}\right)-\left(k_{3}^{2}-k_{o}^{2}\right)\left(k_{e}^{2}-2 k_{3}^{2}\right) H_{0}^{(1)}\left(b k_{o}\right)\right]}\right\} \text {. }
$$

2.4. Current and Input Impedance. Based on King's antenna theory $[18,19]$ on the insulated dipole antenna in a relatively dense medium and rigorous derivation, we can write the current along an insulated antenna in an anisotropic ionosphere as

$$
I(z)=-i \frac{V_{0}}{2 Z_{c}} \frac{\sin k_{l}(l-|z|)}{\cos k_{l} l}
$$

with

$$
Z_{c}=\frac{\sqrt{\mu_{0} / \varepsilon_{\mathrm{II}} k_{l}}}{2 \pi k_{\mathrm{II}}} \ln \frac{b}{a}
$$

where $k_{l}$ and $Z_{c}$ are the wave number in (34) and characteristic impedance of the insulated antenna in the anisotropic ionosphere, respectively. The input impedance for the insulated antenna is

$$
Z_{\text {in }}=\frac{V_{0}}{I(0)}=i 2 \frac{\cos \left(k_{l} l\right)}{\sin \left(k_{l} l\right)} Z_{c} .
$$

\section{Analytical Results}

3.1. Validation of the Analytical Method. In order to verify the accuracy of the analytical method proposed in this paper, the wave number of an insulated antenna in an anisotropic ionosphere is plotted in Figure 2. The length and inner radius of the antenna are $2 l=100 \mathrm{~m}$ and $a=0.01 \mathrm{~m}$, respectively. The plasma frequency is $\omega_{0}=6.6 \times 10^{7} \mathrm{rad} / \mathrm{s}$ and electron cyclotron frequency is $\omega_{H}=8.6 \times 10^{6} \mathrm{rad} / \mathrm{s}$. The working frequency is $f=11.3 \mathrm{kHz}$. The relative permittivity of the insulation layer is $\varepsilon_{\mathrm{II}}=2.32$. $o$ and solid line denote the analytical result in [26] and solution in this paper, respectively. The wave numbers computed with the two methods are in good agreement.

3.2. Wave Number. If not special specified in this following paper, the plasma frequency is $\omega_{0}=7.5 \times 10^{7} \mathrm{rad} / \mathrm{s}$. The electron cyclotron frequency is assumed to be
$\omega_{H}=6.4 \times 10^{6} \mathrm{rad} / \mathrm{s}$. The amplitude of the feeding voltage is $V_{0}=1 \mathrm{~V}$. The radius and conductivity of the good conductor are $a=0.001 \mathrm{~m}$ and $\sigma_{\mathrm{I}}=5.88 \times 10^{7} \mathrm{~S} / \mathrm{m}$, respectively. The relative permittivity of the insulation layer is 1.7 and working frequency of the antenna is $f=3 \mathrm{kHz}$. The lower the working frequency for VLF waves is, the larger the penetration depth of the seawater.

Figure 3 gives effects of the working frequency, normalized insulation thickness, and electric density of the ionosphere on the wave number of an insulated antenna. The real and imaginary parts of the wave number $\operatorname{Re}\left(k_{l}\right)$ and $\mathrm{Im}$ $\left(k_{l}\right)$ represent the attenuation and phase propagation constants. In Figure 3(a), both $\operatorname{Re}\left(k_{l}\right)$ and $\operatorname{Im}\left(k_{l}\right)$ of the insulated antenna increase with the increase of the working frequency and decrease of the thickness of the insulation layer. Figure 3(b) shows $\operatorname{Re}\left(k_{l}\right)$ of the insulated antenna with working frequency $f=20 \mathrm{kHz}$ versus the electric density of the ionosphere. The attenuation propagation constant reaches its peak if the electric density is about $N_{e}=4.6 \times 10^{11} /$ $\mathrm{m}^{3}$.

3.3. Input Impedance. Figure 4 illustrates the driving-point impedance of an open-ended insulted antenna in an anisotropic ionosphere versus the electrical length $\operatorname{Re}\left(k_{l}\right) l$. The real part of the input impedance $\operatorname{Re}\left(Z_{\text {in }}\right)$, that is, the input resistance of the antenna in Figure 4(a) is very sensitive to the insulation thickness and working frequency. Generally speaking, the imaginary part of the driving-point input impedance $\operatorname{Im}\left(Z_{\text {in }}\right)$, that is, the input reactance of the insulated antenna in Figure 4(b) sharply decreases and tends to be smooth as the electrical length increases. Whereas, the driving-point reactance of the open-ended antenna with larger insulation layer $b / a=1.5$ and lower working frequency $f=3 \mathrm{kHz}$ is easier to fluctuate between inductive reactance and capacitive reactance versus the electrical length. $\operatorname{Im}\left(Z_{\text {in }}\right)$ can reach several hundreds of kilo ohms if the length of the insulated antenna is far less than a wavelength.

Because the refractive index of the ionosphere in the VLF band is high, the working wavelength of the VLF waves in 


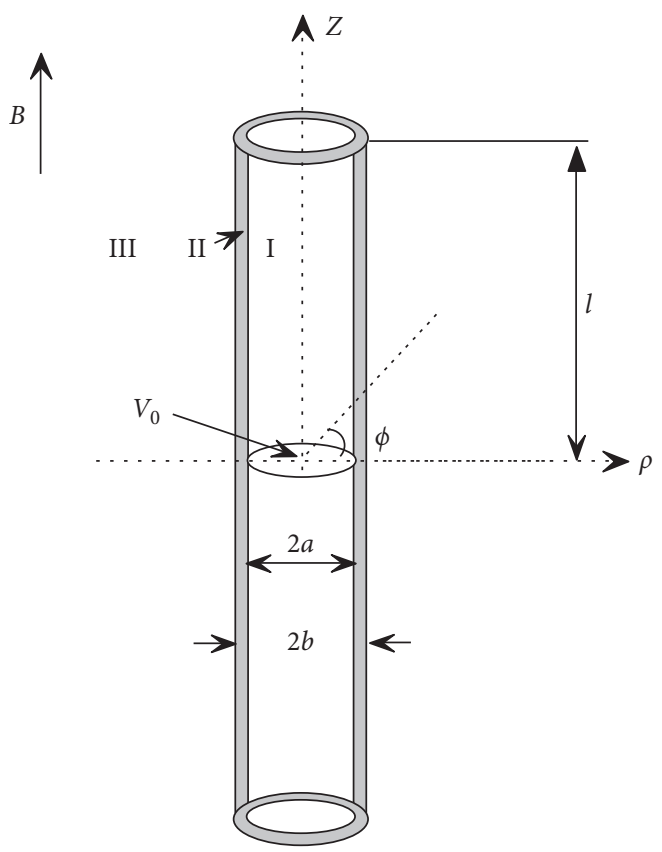

Figure 1: Schematic diagram of an insulated linear antenna in an anisotropic ionosphere.

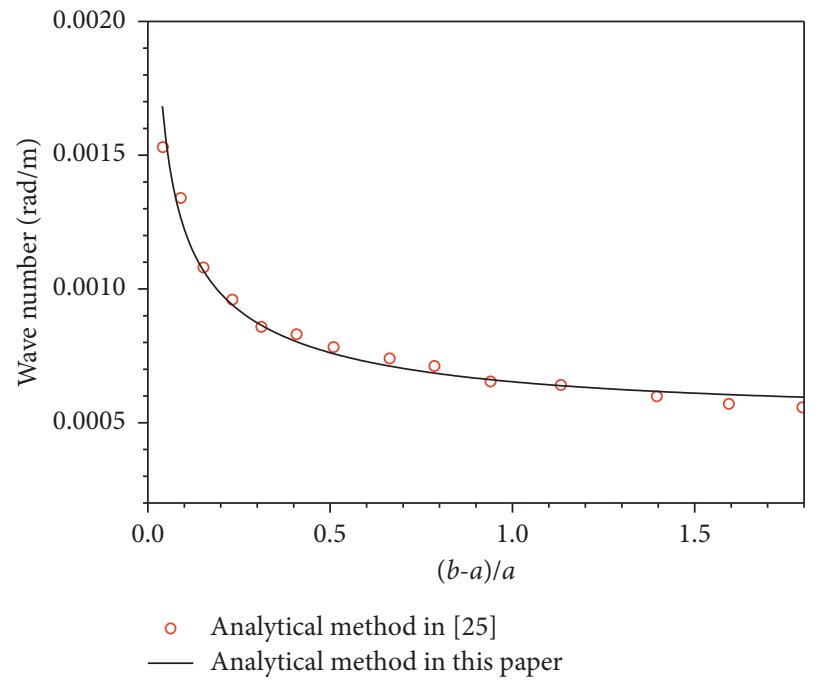

FIgURE 2: Wave number of an insulated antenna in an anisotropic ionosphere versus the normalized insulation thickness.

the ionosphere is shortened. To achieve good radiation efficiency, the input impedance can be obviously optimized by properly selecting the working frequency, insulation thickness, and length of the antenna.

3.4. Current. Figures $5(a)$ and $5(b)$ give the distribution of the normalized current $I / V_{0}$ on the antenna versus the normalized distance from the driving point on the insulated antenna with $a=0.001 \mathrm{~m}, b / a=1.01$, and 2.0 , respectively. We find that the real part of the current decays rapidly along the antenna if the insulation layer is very thin in Figure 5(a). The antenna seems to be a bare antenna whose radiation efficiency seriously reduced by the ionosphere with high conductivity. The imaginary part of the current for the insulated antenna in the anisotropic ionosphere is comparable to that of the real part. By comparing Figure 5(a) with Figure 5(b), one can find that the driving-point current reduces as the thickness of the insulation layer increases, which may further cause the decrease of the radiation efficiency of the antenna and agrees with analytical results in [26]. Whereas the current waveform does not simply exhibit a near-triangular shape and the current distribution of an insulated antenna is sensitive to the insulation thickness, which are contrary to analytical results in [26]. The different effects may be attributed to the complicated relations among the working frequency, plasma frequency, and cyclotron 


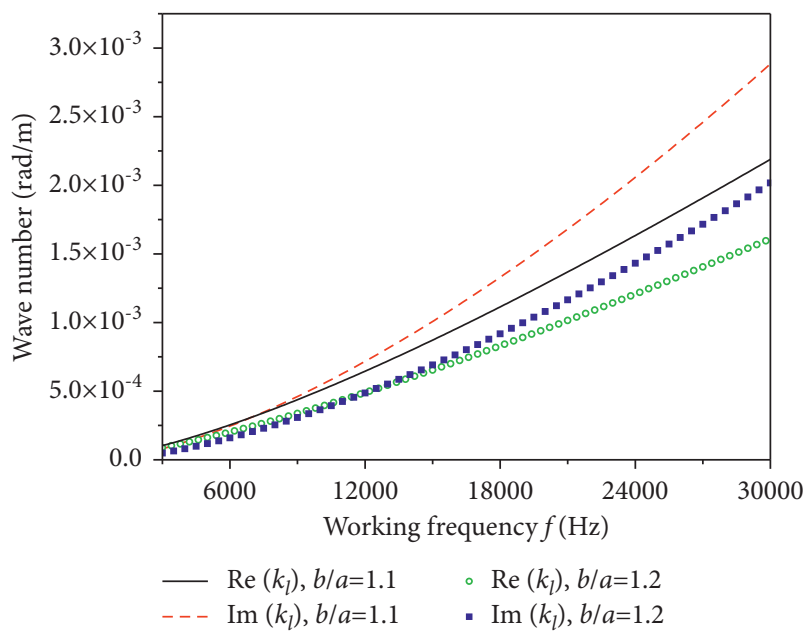

(a)

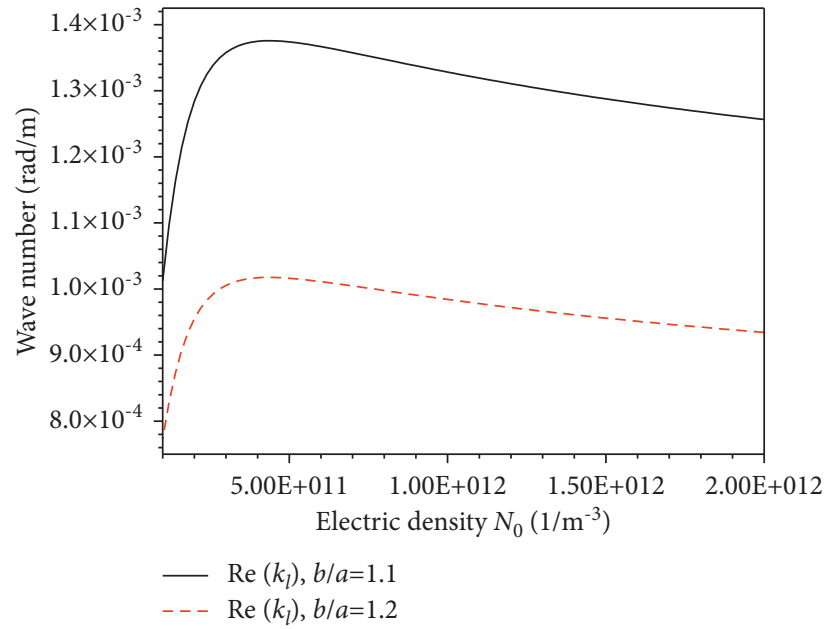

(b)

FIGURE 3: Wave number of an insulated antenna in an anisotropic ionosphere for various normalized insulation thickness (a) versus the working frequency and (b) versus the electric density of the ionosphere.

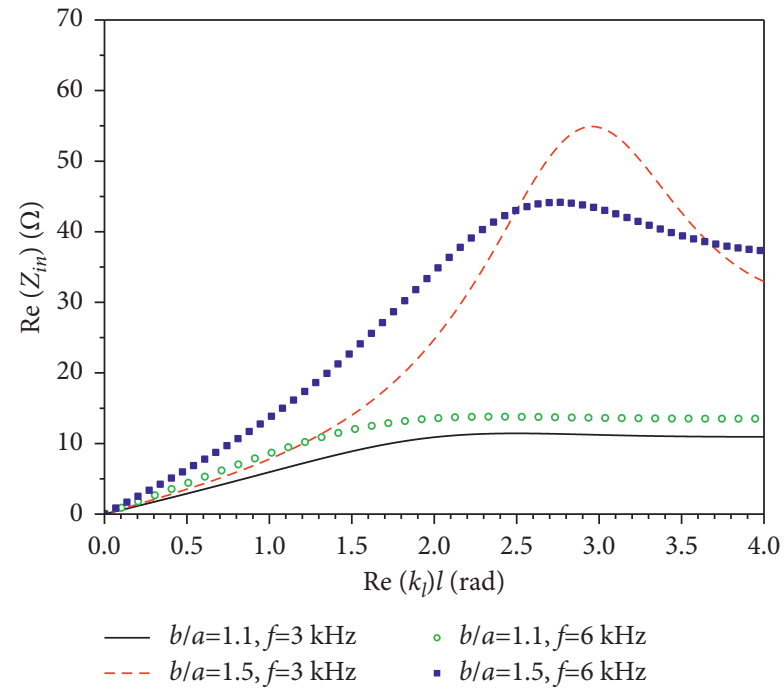

(a)

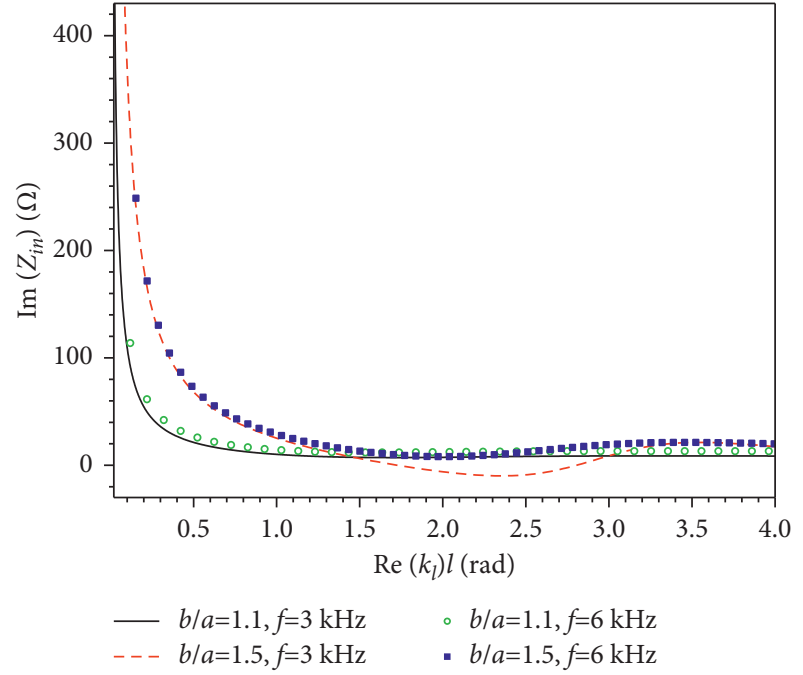

(b)

Figure 4: Input impedance of an insulted antenna in an anisotropic ionosphere for various insulation thicknesses and working frequency. (a) Input resistance and (b) input reactance.

frequency of the ionosphere, which is similar to results in [23]. The sensitivity of the insulated antenna to the surrounding ionosphere is disadvantageous if the antennas are to be used for the communication or telemetry and advantageous if the antennas are used for the diagnostics or exploratory.

Figures 5(c) and 5(d) exhibit the current distribution versus $z / l$ and $\operatorname{Re}\left(k_{l}\right) l$ for different relative permittivity $\varepsilon_{\mathrm{II}}$ of the insulation layer, respectively. In Figure 5(c), the peak value of the driving-point current increases with the increase of $\varepsilon_{\mathrm{II}}$. The real and imaginary parts of the current in Figure 5(d) show nonlinear variation as a function of the electrical length. In order to obtain a larger current moment, the thickness of the insulation layer, the length of the antenna, and relative permittivity of the insulated antenna need to be designed reasonably.

Figure 6 compares effects of medium parameters of the anisotropic ionosphere on the distribution of normalized current on an insulated antenna with $b / a=1.5$ in an anisotropic ionosphere. The medium parameters, such as the electric density and geomagnetic field, which can be obtained from the international reference ionosphere model and international geomagnetic reference field model, for the anisotropic F2 layer of the ionosphere are influenced by the latitude, longitude, height, diurnal variation, seasonal variation, etc. 


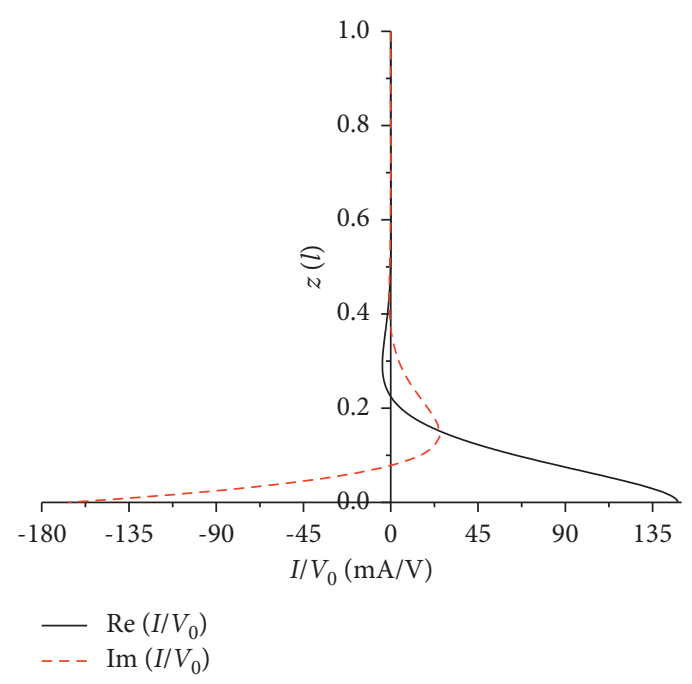

(a)

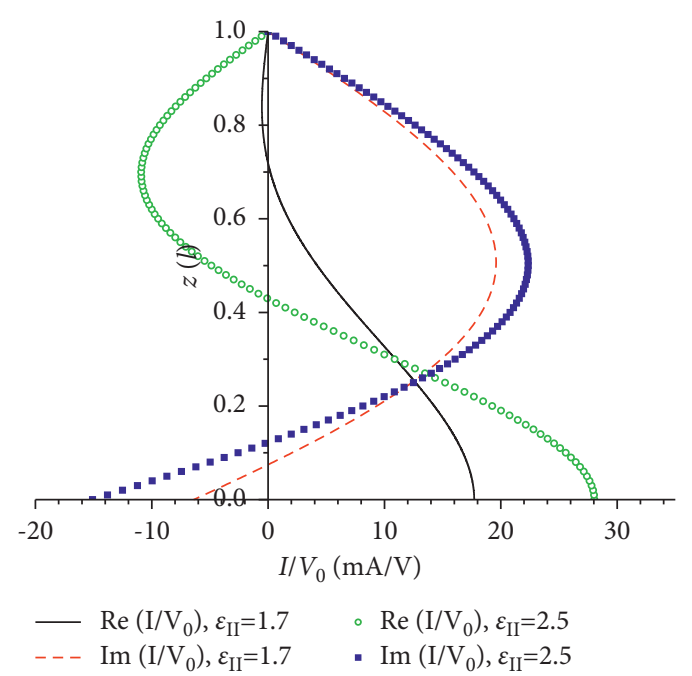

(c)

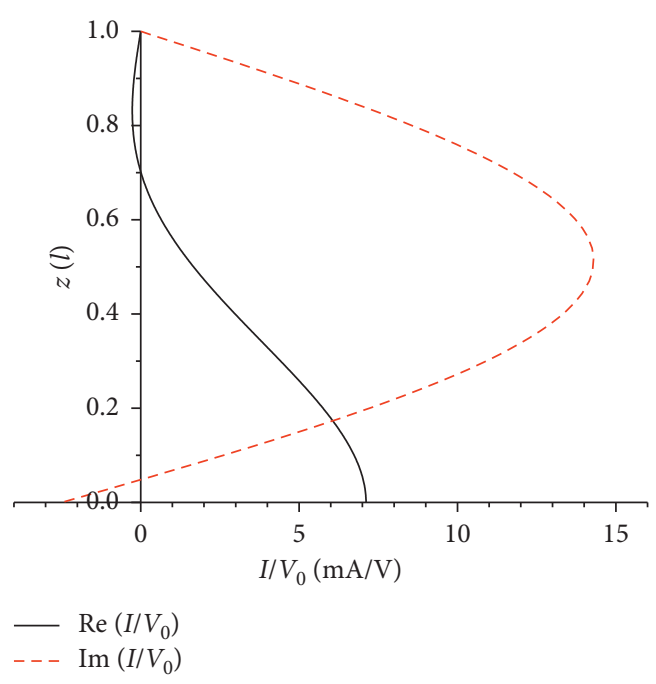

(b)

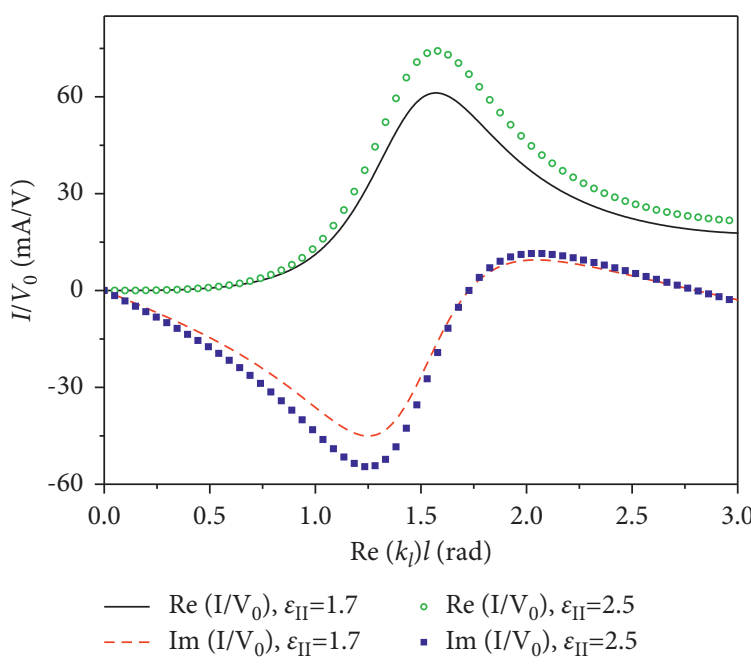

(d)

FiguRE 5: Real and imaginary parts of normalized current on an insulated antenna in an anisotropic ionosphere. (a) $b / a=1.01$, Re $\left(k_{l}\right)$ $l=10.75$, (b) $b / a=2.0, \operatorname{Re}\left(k_{l}\right) l=3.25$, (c) $b / a=1.5$ versus $z / l$, and (d) $b / a=1.5$ versus $\operatorname{Re}\left(k_{l}\right) l$.

In Figures 6(a) and 6(b), the driving-point current is nearly affected by the electric density. Whereas the higher the electric density of the ionosphere is, the larger the amplitude of the current along the radiating antenna is. That is, the current on the antenna at a certain location, which is stationary relative to the Earth, of the ionosphere in the daytime is larger than that at night due to higher electric density during the day and lower electric density during the night. Besides the time, the current on the antenna varies with the location where the antenna is replaced because of the changeable electric density.
As shown in Figures 6(c) and 6(d), the magnitude of the geomagnetic field has a big effect on the current distribution of the insulated antenna. If the relative permittivity, which contains the gyromagnetic frequency as a function of the magnitude of the geomagnetic field, of the anisotropic ionosphere differs, the variation of input impedance and propagation modes of the insulated antenna in different geomagnetic environment influences the current distribution. The amplitude of driving-point current fluctuates to a limited extent as the magnitude of the geomagnetic field and electrical length of the antenna increase. 

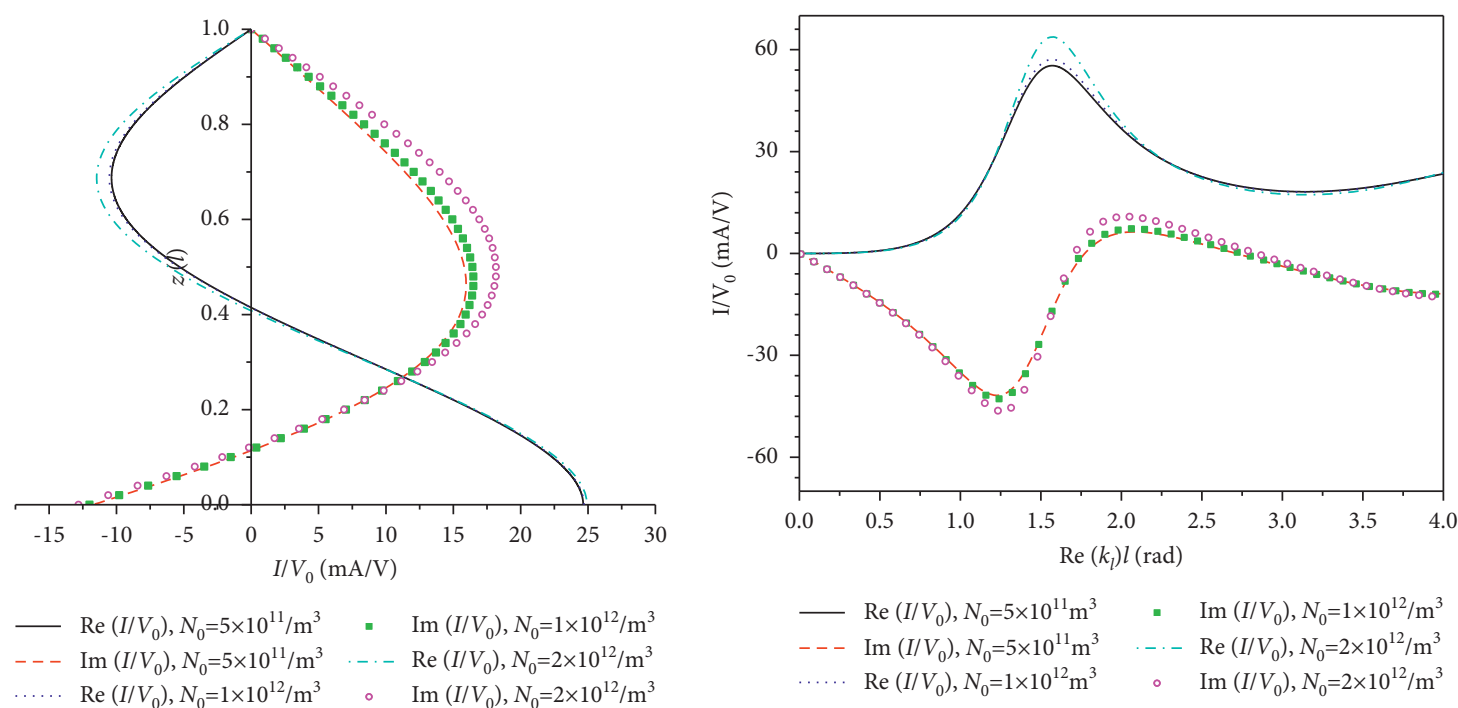

(a)

(b)

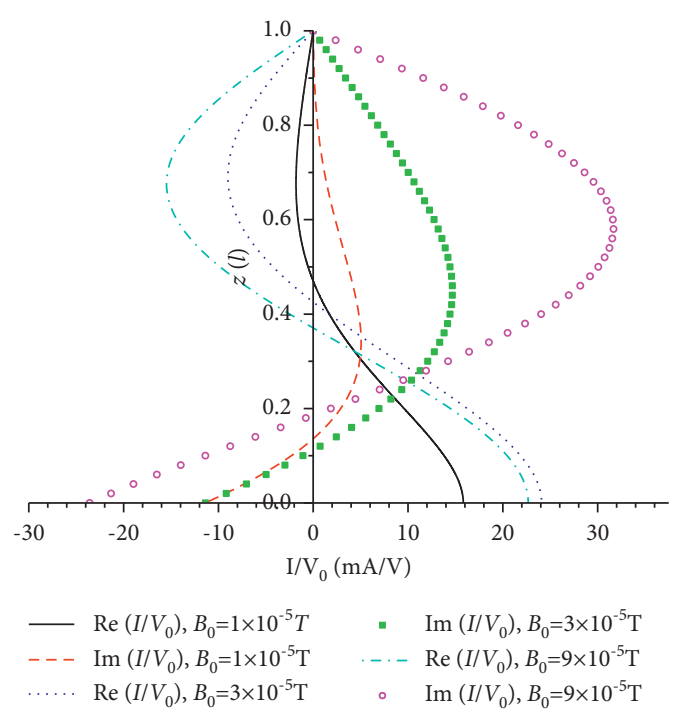

(c)

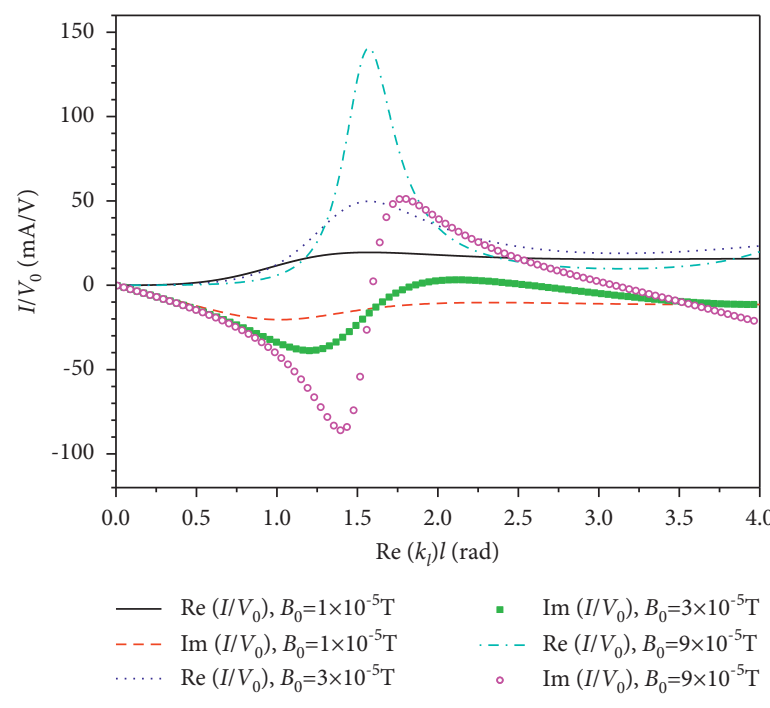

(d)

FiguRE 6: Real and imaginary parts of the normalized current on an insulated antenna in an anisotropic ionosphere for different electric densities and magnitudes of geomagnetic field. (a) Effects of electric densities versus $z / l$, (b) effects of electric densities versus Re $\left(k_{l}\right) l$, (c) effects of geomagnetic field versus $z / l$, and (d) effects of geomagnetic field versus $\operatorname{Re}\left(k_{l}\right) l$.

\section{Conclusion}

A novel method is proposed to calculate near-field characteristics of a VLF insulated antenna in an anisotropic ionosphere in this paper. To solve the wave number, the eigenvalue equation for the circularly layered structure containing an infinite homogeneous ionosphere is derived. Based on these results, King's antenna theory is developed for an infinite insulated antenna located in the anisotropic ionosphere. Analytical results show that near-field characteristics of the insulated antenna in the ionosphere, which are similar to those in the air, are sensitive to the working frequency, electrical length, relative permittivity, and thickness of the insulation layer. The current distribution of the insulated antenna is more easily influenced by the amplitude of the geomagnetic field than electric density for the F2 layer of the anisotropic ionosphere. The medium parameters which are affected by the location and time of the ionosphere, where the antenna replaces, need to be adequately considered. The reported findings are believed to provide guidelines for the antenna design of the space-borne communication system. 


\section{Conflicts of Interest}

The authors declare that they have no conflicts of interest.

\section{Acknowledgments}

This work was supported in part by the National Natural Science Foundation of China (Grant nos. 61971351 and 61401361) and the Fund of the National Key Laboratory of Electromagnetic Environment (Grant nos. 6142403 and 202102010).

\section{References}

[1] J. K. Harrison and C. C. Rupp, "Orbiting transmitter and antenna for spaceborne communications at ELF/VLF to submerged submarines," in Proceedings of the AGARD Conference, vol. 592, pp. 33.1-33.14, Berlin, Germany, 1993.

[2] W. H. Hou, Q. L. Zhang, J. B. Zhang, L. Wang, and Y. Shen, "A new approximate method for lightning-radiated ELF/VLF ground wave propagation over intermediate ranges," International Journal of Antennas and Propagation, vol. 2018, Article ID 9353294, 10 pages, 2018.

[3] C. L. Chang, G. Bergeron, A. D. Drobot, K. Papadopoulos, S. Riyopoulos, and E. Szuszczewicz, Theory and Modeling in Support of Tether, NASA Marshall Space Flight Center, Huntsville, AL, USA, 1999.

[4] N. A. Armand and Y. P. Semenvo, "Experimental researches in the ionosphere of the earth of the radiation of loop antenna in a range VLF waves, installed onboard the orbital complex "progress-28"-“Souz TM-2"," Radio-Electronics, vol. 33, pp. 2225-2233, 1988.

[5] T. He, H. R. Zeng, and K. Li, "VLF current distribution and input impedance of an arbitrarily oriented linear antenna in a cold plasma," IEEE Access, vol. 7, pp. 80861-80869, 2019.

[6] Y. L. Yan, C. Liu, H. N. Wu, and Y. H. Dong, "Non-foster matching circuit design via tunable inductor for VLF receive loop antennas," International Journal of Antennas and Propagation, vol. 2017, Article ID 6198686, 10 pages, 2017.

[7] M. Parrot, "The micro-satellite DEMETER," Journal of Geodynamics, vol. 33, no. 4-5, pp. 535-541, 2002.

[8] H. J. Kramer, Observation of the Earth and its Environment: Survey of Missions and Sensors, Springer-Verlag, Berlin, Germany, 2002.

[9] L. G. Abraham, Extensions of the magnetoionic theory for radio-wave propagation in the ionosphere including antenna radiation and plane-wave scattering, Thesis ( $\mathrm{PhD})$, Cornell University, Ithaca, NY, USA, 1953.

[10] Y. Chow, "A note on radiation in a gyro-electric-magnetic medium-an extension of bunkin's calculation," IRE Transactions on Antennas and Propagation, vol. 10, no. 4, pp. 464-469, 1962.

[11] H. H. Kuehl, "Electromagnetic radiation from an electric dipole in a cold anisotropic plasma," Physics of Fluids, vol. 5, no. 9, pp. 1095-1103, 1962.

[12] H. Hodara, "Radiation from a gyro-plasma sheathed aperature," IEEE Transactions on Antennas and Propagation, vol. 11, no. 1, pp. 2-12, 1963.

[13] P. C. Clemmow, "The theory of electromagnetic waves in a simple anisotropic medium," Proceedings of the Institution of Electrical Engineers, vol. 110, no. 1, pp. 101-106, 1963.

[14] C. Edward, Electromagnetic Theory and Antennas, Macmillan, London, UK, 1963.
[15] R. Mittra and G. A. Deschamps, Field Solution for a Dipole in an Anisotropic Medium, Kluwer Academic Publishers, Dordrecht, Netherlands, 1963.

[16] T. N. C. Wang and T. F. Bell, "On VLF radiation resistance of an electric dipole in a cold magnetoplasma," Radio Science, vol. 5, no. 3, pp. 605-610, 1970.

[17] T. N. C. Wang and T. F. Bell, "Electric dipole radiation at VLF in a uniform warm magneto-plasma," Revue de Physique Appliquee, vol. 7, no. 1, pp. 11-20, 1972.

[18] R. W. P. King and G. S. Smith, Antennas in Matter: Fundamentals. Theory and Applications, MIT Press, Cambridge, MA, USA, 1981.

[19] T. T. Wu, R. W. P. King, and D. V. Giri, "The insulated dipole antenna in a relatively dense medium," Radio Science, vol. 8, no. 7, pp. 699-709, 1973.

[20] K. Balmain, "The impedance of a short dipole antenna in a magnetoplasma," IEEE Transactions on Antennas and Propagation, vol. 12, no. 5, pp. 605-617, 1964.

[21] P. Nikitin and C. Swenson, "Impedance of a short dipole antenna in a cold plasma," IEEE Transactions on Antennas and Propagation, vol. 49, no. 10, pp. 1377-1381, 2001.

[22] C. Chen and S. Seshadri, "Infinite insulated cylindrical antenna in a simple anisotropic medium," IEEE Transactions on Antennas and Propagation, vol. 14, no. 6, pp. 715-726, 1966.

[23] J. Galejs, "Impedance of a finite insulated cylindrical antenna in a cold plasma with a longitudinal magnetic field," IEEE Transactions on Antennas and Propagation, vol. 14, no. 6, pp. 727-736, 1966.

[24] A. V. Kudrin, E. Y. Petrov, G. A. Kyriacou, and T. M. Zaboronkova, "Insulated cylindrical antenna in a cold magnetoplasma," Progress in Electromagnetics Research, vol. 53, pp. 135-166, 2005.

[25] T. He, X. W. Zhang, W. Y. Pan, and K. Li, "Current distribution and input impedance of a VLF linear antenna in an anisotropic plasma," IEEE Transactions on Antennas and Propagation, vol. 67, no. 3, pp. 1519-1526, 2019.

[26] H. R. Zeng, T. He, and K. Li, "Current distribution and input impedance of an insulated linear antenna in an anisotropic plasma," IEEE Transactions on Antennas and Propagation, vol. 68, no. 4, pp. 2541-2549, 2020.

[27] M. L. Bouknia, C. Zebiri, D. Sayad et al., "Theoretical study of the input impedance and electromagnetic field distribution of a dipole antenna printed on an electrical/magnetic uniaxial anisotropic substrate," Electronics, vol. 10, p. 1050, 2021.

[28] M. L. Bouknia, C. Zebiri, D. Sayad et al., "Analysis of the combinatory effect of uniaxial electrical and magnetic anisotropy on the input impedance and mutual coupling of a printed dipole antenna," IEEE Access, vol. 9, pp. 84910-84921, 2021.

[29] Q. D. Chen, R. Liu, Y. Liu, X. Huang, W. P. Xu, and W. M. Zhen, "LF/VLF electromagnetic pulse measurement system," Chinese Journal of Radio Science, vol. 35, no. 5, pp. 791-798, 2020.

[30] J. X. Sun, H. Y. Peng, and Q. L. Li, "Analysis of disturbance of VLF wave to low ionosphere based on full wave method," Chinese Journal of Radio Science, vol. 36, no. 4, pp. 619-629, 2021.

[31] K. Li and W. Y. Pan, "Propagation of VLF electromagnetic waves penetrating the lower ionosphere," Indian Journal of Radio \& Space Physics, vol. 28, no. 2, pp. 87-94, 1999.

[32] I. V. Kuzichev, "On the ionospheric propagation of VLF waves generated by currents in the lower ionosphere," Journal of Atmospheric and Solar-Terrestrial Physics, vol. 179, pp. 134-148, 2018. 
[33] W. Y. Pan, Long Wave Ultra Long Wave Propagation, University of Electronic Science and Technology Press, Chengdu, China, 2004.

[34] G. K. Parks, Physics of Space Plasmas: An Introduction, Westview Press, Boulder, CO, USA, 2004.

[35] H. L. Xu, T. T. Gu, Y. Zhu, H. C. Yin, and L. X. Yang, "The mode-conversion coefficient of ELF wave propagation due to land-to-sea transition," International Journal of Numerical Modeling: Electronic Networks, Devices and Fields, vol. 34, no. 6, p. e2820, 2020. 